\title{
Perspectives for the pharmacological treatment of overactive bladder syndrome
}

\author{
Andrzej Wróbel ${ }^{1}$, Tomasz Kluz ${ }^{2}$, Grzegorz Surkont ${ }^{3}$, Edyta Wlaźlak ${ }^{3}$, \\ Paweł Miotła ${ }^{1}$, Katarzyna Skorupska ${ }^{1}$, Tomasz Rechberger ${ }^{1}$ \\ ${ }^{1}$ Second Department of Gynecology, Medical University of Lublin, Poland \\ 2Department of Obstetrics and Gynecology, Fryderyk Chopin University Hospital No 1, Faculty of Medicine, \\ Rzeszow University, Poland \\ ${ }^{3}$ Department of Operative Gynecology and Gynecologic Oncology, $1^{\text {st }}$ Department of Gynecology and Obstetrics, \\ Medical University of Lodz, Poland
}

\begin{abstract}
It has been found that antimuscarinic drugs, viewed as the "gold standard" in overactive bladder syndrome (OAB) treatment, have an unsatisfactory tolerance profile and limited clinical effectiveness. This fact has given a clear impetus to search for new options in $O A B$ pharmacotherapy. The conducted pre-clinical trials have led to the development of new solutions for the treatment of $O A B$, which stand a good chance of being applied in clinical practice. The said compounds are characterised by higher receptor and organ specificity than currently used medications.
\end{abstract}

Key words: overactive bladder syndrome, pharmacotherapy, detrusor overactivity

Ginekologia Polska 2017; 88, 9: 504-508

\section{INTRODUCTION}

The review of the current state of knowledge on the perspectives for the pharmacological treatment of $O A B$ is presented below. It focuses on the mechanisms of action of new compounds at the same time indicating the possibilities for their application in specific groups of patients. Due to its complex etiopathogenesis, OAB is seen as "a disease of symptoms," and it seems justified to expect that the future pharmacotherapy of this syndrome should be tailored to the individual needs of a given patient.

\section{NEW ANTIMUSCARINICS}

Due to their proven effectiveness, anticholinergics are still the subject of extensive studies which focus on new medications with regard to their receptor and organ selectivity, the development of new methods of drug administration and combined therapies using existing compounds.

Imidafenacin (KRP-197, ONO8025) is a newly-synthesised antimuscarinic agent registered for $O A B$ treatment, and launched on the Japanese and Taiwanese markets. It shows high selectivity for $M_{3}$ and $M_{1}$ receptors, and low for $M_{2}$ receptor, along with organ selectiveness in relation to the urinary bladder. The drug inhibits bladder contractibility acting as an $M_{3}$ receptor antagonist, and modulating the release of acetylcholine from the prejunctional facilitatory $M_{1}$ receptors. The meta-analysis of five randomised clinical trials devoted to the evaluation of the safety profile and efficacy of imidafenacin, which covered 1253 patients, did not show any significant differences in the number of urge incontinence episodes or the mean number of voidings per day, when compared to propiverine or solifenacin. Also, differences in $O A B$ index values (OABSS) were not found. Imidafenacin proved to have a beneficial effect on nocturia and on the polyuria index. Such results, however, were not obtained with regard to solifenacin. When compared with propiverine, solifenacin and fesoterodine, they produced lower number of constipations and intensification of dry-mouth symptoms. The duration of dry-mouth symp- 
toms following the administration of imidafenacin proved to be much shorter than with other anticholinergics. It is very important that imidafenacin does not affect the cognitive function in elderly patients and does not induce cardiovascular symptoms typical for anticholinergics, such as increased heart rate or the prolongation of the QT interval on ECG records. Interestingly, imidafenacin, unlike other antimuscarinics, does not increase the plasma concentration of digoxin administered at the same time as imidafenacin [1].

Tarafenacin (SVT-40776) is a new quinuclidinol derivative with high $\mathrm{M}_{3}$ receptor selectivity (an antagonist) and low inhibition potential in relation to the submandibular gland. It shows a 200 times higher selectivity for $M_{3}$ receptors than for $M_{2}$ receptors. The second-phase trials, which comprised 235 people receiving tarafenacin in a dose of $0.2 \mathrm{mg}$ or $0.4 \mathrm{mg}$, showed a considerable drop in the number of voidings per day in the group receiving $0.4 \mathrm{mg}$ of the drug for 12 weeks, when compared to the placebo group. The three groups displayed no differences in the frequency of urinary urgency. Dose-response correlation, in turn, was found with regard to the increase of dry-mouth and visual disorders while the increase in the dose did not exacerbate constipations. Tarafenacin is now administered as part of the third phase of the clinical trials. The initial results seem to indicate that the action of the drug begins quickly, reaching maximum blood concentration within $4 \mathrm{~h}$ after swallowing, and $\mathrm{T}_{1 / 2}$ - within $35 \mathrm{~h}$, as a result of which it can be taken once a day. No significant cardiovascular adverse events have been noted so far [2].

Afacifenacin (SMP-986) is another anticholinergic agent which is now administered in the second phase of the clinical trials. It has a unique mechanism of actions. In addition to blocking $\mathrm{M}$ receptors, afacifenacin proves to have a blocking effect on the sodium channels, leading to the inhibition of afferent fibres which supply the urinary bladder. The clinical effect of this drug include a reduction in pollakiuria and urge incontinence, and an increase in voiding volume. The results of the currently conducted trials indicate that, when compared to other antimuscarinics, the drug displays less intensified adverse effects, which may stem from the high receptor selectivity of this compound.

\section{NEW COMBINATIONS OF DRUGS}

Adverse effects, typical of the pharmacotherapy involving anticholinergics (including especially dry mouth), have given a clear impetus for work on a new strategy of complex therapy, including the combined application of pilocarpine - a quick-acting muscarinic receptor antagonist, characterised by high organ selectivity for salivary glands (it stimulates their secretionary function), and an antimuscarinic agent. Tolenix (THVD-201) is a combination of tolterodine IR (2 mg) and pilocarpine ER (9 mg). 138 people randomly assigned to one of the three groups - receiving tolenix, tolterodine or the placebo - were subjected to observations. When compared to the placebo group, the other two displayed a reduction in $O A B$ symptoms in the form of voiding frequency reduction and a lower number of urge incontinence episodes, but they suffered from strong dry-mouth effect. Importantly, significant drops in dry mouth, difficulty in swallowing, chewing and falling asleep in the Tolenix group were noted, when compared to the patients receiving tolterodine IR. Urge incontinence episodes were found to be slightly more frequent in the group receiving Tolenix than in the patients receiving tolterodine. The Tolenix group had low visual analogue scores for dry mouth. Salivary gland secretion did not change after $3 \mathrm{~h}$ of the administration of Tolenix, but it became significantly lower after the administration of tolterodine IR. The medication is currently subjected to the third phase of clinical trials [3].

\section{$\beta_{3}$ RECEPTOR AGONISTS}

Solabegron (GW427353) is a new $\beta_{3}$ receptor agonist, currently undergoing clinical tests. The previous in vitro tests on the urinary bladder sections showed that this medication caused an $82 \%$ relaxation of the bladder tissue. Also, the degree of selectivity of solabegron for $\beta_{1}, \beta_{2}$ and $\beta_{3}$ receptors was determined. This selectivity was measured by observing the CAMP increase. Following the solabegron administration, the CAMP increased by $90 \%$ in the cells containing $\beta_{3}$ receptors, and by only $<10 \%$ in those cells which had $\beta_{1}$ and $\beta_{2}$ receptors. Interestingly, selective antagonists of $\beta_{1}$ and $\beta_{2}$ receptors lacked the relaxant potential of solabegron. In vitro tests also showed that solabegron reinforced the relaxant effect induced by oksybutynin, tolterodine, or solifenacin, and is a much stronger relaxant agent than mirabegron. At present, the second phase of the clinical trials is conducted, aiming at assessing this interaction. In vivo tests have proven that the drug increases voiding volume by $22 \%$. The second phase of the trials devoted to the examining of its effectiveness covered 258 patients who were divided into three groups - a placebo group and two groups receiving 50 and $125 \mathrm{mg}$ of the medicine, respectively, every $12 \mathrm{~h}$. After 8 weeks of treatment a significant decrease in the number of urge incontinence episodes was noted in both study groups, with the same degree of dry mouth and constipation as in the placebo group. A drop in the voiding frequency, and an increase in voiding volume and volume threshold to elicit nonvoiding contractions (VTNVC) values, in the urodynamic test were noted in the group of patients receiving higher doses of the drug. No significant changes were found with regards to arterial pressure, or alarming symptoms visible on ECG [4].

It is believed that $\beta_{3}$ receptor agonists might become second-line medications in those OAB patients who poorly 
respond to treatment with antimuscarinics and do not tolerate them, or when there is a risk of urine retention.

\section{NEW STRATEGIES FOR BOTULINUM TOXIN ADMINISTRATION}

While administering the botulinum toxins clinicians usually avoid the urinary bladder triangle due to the risk of muscle irritation and vesicoureteral reflux. A comparative study of cases in which the toxin was administered within and outside the triangle, as well as to both those sites, showed no differences in the effectiveness of the treatment or adverse effects frequency. In one of the trials conducted in a group of 22 people, the injections to the triangle were found to be more effective when compared to the injections outside the bladder triangle. The patients were given 500 units of neurotoxin in $20 \mathrm{~mL}$, in 20 injections. A primary final point was the assessment of the OAB index. OABSS improved from 22.4 to 8.7 in the group which was injected inside the triangle and from 22.7 to 13.4 in the group receiving neurotoxin outside this area. No differences in PVR were noted.

Clinical trials are also conducted with the aim of evaluating the effectiveness of the intravesical instillation of botulinum toxin combined with liposomes, which is thought by the researchers to improve the transport of neurotoxin through the urothelium to the detrusor. The trials involved the administration of lipotoxins containing $80 \mathrm{mg}$ of liposomes and 200 units of A botulinum toxin. The initial results of the experiments showed that the number of urinary urgency and pollakiuria episodes were reduced after one or three months following the instillation. Changes in PVR or increases in urinary infection frequency were not noted. The work aimed at determining the depth of lipotoxin penetration have not been concluded yet, so it is possible that the mechanisms of its action are slightly different from those of botulinum toxin injections, due to the transport limitations from the urothelium to deeper layers of the bladder. This neurotoxin has a high molecular weight (150 KDa), and, if it is not injected, it might not be able to reach the afferent fibres lying below the urothelium [5].

\section{ANTIDEPRESSANTS}

The patients with $O A B$ frequently suffer from various mental disorders, among which depression is the most common. It has been proven that duloxetine produces positive therapeutic effects, not only in SUI treatment, but it can also be seen as a drug of choice in MS patients experiencing both depression and OAB symptoms. Its effectiveness has been confirmed during the treatment of patients with both $O A B$ wet and $O A B$ dry. Tricyclic antidepressants (imipramine or amitriptyline) which increase NA levels through the inhibition of its reuptake are used as a second-line treatment (off label) in people with OAB resistant to antimuscarinic therapy.
Preclinical trials have indicated that the beneficial effect which duloxetine has on the OAB symptoms stems from the inhibition of sensory afferents by the serotonegic system, and also from modulation by the serotonin and noradrenaline micturition centre in the sacra spinal cord. The results of clinical trials have proven that this drug decreases the number of frequency and urgency episodes, and improves the quality of life scores, while leaving the mean voided volume unchanged. No adverse effects have been found in relation to the heart rate, blood pressure or arrhytmogenic tendencies in EEG. Its clinical efficacy has proven to exceed the placebo effects in $O A B$ patients due either to detrusor overactivity (DO) or sensory urgency, and in MUI patients. Thus, due to the proven clinical effectiveness in treating depression, it seems that duloxetine can become a drug of choice in patients with depression and coexisting OAB symptoms [6].

\section{ANTIEPILEPTIC DRUGS}

Gabapentin was first synthesised as an antiepileptic drug. Its mechanisms of action are based on blocking the sodium channels and the modulation of GABAergic neurotransmission. It is currently under clinical trials in the terms of its effectiveness in OAB pharmacotherapy. The results to date indicate that gabapentin has positive effects on $O A B$ symptoms in neurogenic bladder patients, and in patients resistant to treatment with anticholinergics. Cystometric measurements have shown the reduction in the urodynamic symptoms of DO. It has also been found that the combined application of gabapentin and NSAID or amitryptyline has positive effects on DO symptoms in people with the bladder pain syndrome. Pregabin and lamotrigine are drugs with structures similar to gabapentin, and it has turned out that their administration to patients with neurogenic DO could give positive results [7].

\section{TACHYKININ RECEPTORS}

The results of the preclinical trials have proven that the contractions in the human detrusor muscle induced by tachykinins are mediated by the $\mathrm{NK}_{2}$ receptors. The stimulation of these receptors by tachykinins released from afferent endings leads to the lowering of the voiding threshold, and, as a consequence, to the detrusor overactivity and the occurrence of phase contractions. It might suggest that tachykinins participate in supraspinal micturition control, which makes NK receptors located in the spinal cord a potential target of OAB pharmacotherapy. The results of the clinical trials have proven that the antagonists of $\mathrm{NK}_{2}$ receptors inhibit hyperreflexia in bacterial cystitis and in cases of spinal cord injuries. This opens up a potential for drug application in patients with MS and spinal cord injuries accompanied by $\mathrm{OAB}$ symptoms. One of the antagonists to $\mathrm{NK}_{1}$ receptors 
which can penetrate the blood-brain barrier is aprepitant, which is now used to treat nausea in patients undergoing chemotherapy. Randomised clinical trials have shown that this drug has a positive effect on urinary frequency and urgency episodes. Another antagonist to $\mathrm{NK}_{1}$ receptors - serlopitant - has also been tested with regard to its effectiveness. It has proven to reduce the number of urgency episodes, whereas its clinical efficacy appears lower than that of tolterodine ER [8].

\section{TRPV $_{1}$ RECEPTORS}

Urinary bladder and urethra are characterised by a high density of TRPV 1 receptors which take part in pain perception and bladder contractile activity. A particularly high expression of these receptors is found in the urothelium and demyelinised bladder C fibres. TRPV 4 receptors, in turn, act as mechanoreceptors and play an important role in the regulation of bladder excitability and contractibility. Preclinical trials have shown that the antagonists of $\mathrm{TRPV}_{4}$ receptor decrease the voiding frequency. It has also been found that the selective agonists of TRPV receptors (capsaicin and resiniferatoxin), when administered as a single dose, cause the depolarisation of afferent fibres containing TRPV ${ }_{1}$ receptors. If they are administered multiple times, they decrease sensitivity, impair the functions of and completely damage the endings of these neurons. This produces a clinical effect in the form of an increase in functional bladder volume. TRPV 1 receptor antagonists also constitute an interesting alternative in $O A B$ pharmacology. They have been found to block the release of ATP from the urothelium induced by the bladder wall stretching, and at the same time they do not cause adverse events typical for TRPV ${ }_{1}$ agonists, and inhibit detrusor contractions induced by the administration of cyclophosphamide. Capsazepine is representative for this group of compounds and is currently subjected to clinical tests [9].

\section{CANNABINOID RECEPTORS}

The presence of cannabinoid receptors $\left(\mathrm{CB}_{1}, \mathrm{CB}_{2}\right)$ was detected in many structures of CNS. They play an important role in voiding cycle regulation. $\mathrm{CB}_{1}$ receptors (but not $C B_{2}$ receptors) were also found in the neurons of the dorsal root of the spinal nerve. A particularly high density of $\mathrm{CB}_{1}$ and $\mathrm{CB}_{2}$ receptors was detected in the urothelium, and was much higher than the level found in the detrusor muscle. Preclinical trials have proven that the stimulation of cannabinoid receptors leads to a reduction in bladder contractile activity. The stimulation of $\mathrm{CB}_{1}$ receptors has also been found to inhibit DO induced by the intravesical administration of NGF. This reaction could be reversed by the application of the selective antagonist. Synthetic analogues of these compounds have proven to reduce DO symptoms induced by nociceptive stimuli. The clinical trials have con- firmed the high effectiveness of cannabis extract containing delta-9-tetrahydrocannabinol in multiple-sclerosis patients with urge incontinence. Thus, it seems that $C_{1}$ (and probably also $\mathrm{CB}_{2}$ ) receptors can constitute a potential target for OAB pharmacotherapy [10].

\section{VITAMIN D 3 RECEPTORS}

Vitamin $D_{3}$ receptors were found in the cells of urothelium and the smooth muscle cells of the detrusor, and also in the urethra. Preclinical trials have shown that vitamin analogues have a relaxant effect on the bladder through the upregulation of L-type $\mathrm{Ca}^{2+}$ channel and ROCK inhibition. It was confirmed by the results of the clinical trials in which the authors noted that the administration of elocalcitol in people with $\mathrm{OAB}$ symptoms and features of $\mathrm{DO}$, as observed during the urodynamic tests, resulted in a reduction in the number of incontinence episodes, and a subjective improvement in the patient's health condition. However, its effects on the DO features present in cystometry were not revealed. In turn, the application of elocalcitol in patients with idiopathic DO proved to increase the voiding and the bladder volumes at the first desire to void. This compound administered to people suffering from benign prostatic hyperplasia led to a reduction in urgency and daytime and night-time frequency. In turn, another vitamin $D_{3}$ analogue (maxacalcitol) weakened the positive effects the ROCK inhibitors had on DO. At the same time, it was noted that doses of this analogue, which were ineffective as a monotherapy, potentialised the action of oxybutynin chloride and amlodipine besylate. The results of clinical trials have shown that the therapy with vitamin D analogues is welltolerated and could be used in the pharmacotherapy of OAB wet, especially in people who do not tolerate treatment with antimuscarinics, or when such therapy proves ineffective [11].

\section{OPIOID RECEPTORS}

Opioids seem to affect voiding, both through their impacts on CNS and via receptors localised in the nervous system. The intravenous administration of tramadol increases the threshold micturition pressure and voiding volume. Used as a prolonged release agent, the drug has proven more effective than the placebo in its reduction of the number of urge-incontinence episodes. When administered epidurally, tramadol leads to an increase in bladder volume and susceptibility, without affecting the voiding phase. Also, the agonist of the opioid receptors $\left(\mathrm{OP}_{4}\right)$ - nociceptin is currently subjected to clinical trials. The results so far have shown that its intravesical instillation in patients with neurogenic bladder induces bladder volume increase, which might suggest that this group of compounds can become an interesting alternative to vanilloids $[12,13]$. 


\section{SUMMARY}

The obtained results from both preclinical and clinical trials identified new pharmacological options of $O A B$ therapy which stand a good chance of being applied in clinical practice. It seems that they will be tailor-made and based on the pathophysiological mechanisms that induce OAB symptoms.

\section{REFERENCES}

1. Leone Roberti Maggiore U, Scala C, Venturini PL, et al. Imidafenacin for the treatment of overactive bladder. Expert Opin Pharmacother. 2013; 14(10): 1383-1397, doi: 10.1517/14656566.2013.796930, indexed in Pubmed: 23641864.

2. Song $\mathrm{M}, \mathrm{Kim} \mathrm{JH}$, Lee KS, et al. The efficacy and tolerability of tarafenacin, a new muscarinic acetylcholine receptor $\mathrm{M} 3$ antagonist in patients with overactive bladder; randomised, double-blind, placebo-controlled phase 2 study. Int J Clin Pract. 2015; 69(2): 242-250, doi: 10.1111/ijcp.12540, indexed in Pubmed: 25363415.

3. Dmochowski RR, Staskin DR, Duchin K, et al. Clinical safety, tolerability and efficacy of combination tolterodine/pilocarpine in patients with overactive bladder. Int J Clin Pract. 2014; 68(8): 986-994, doi: 10.1111/ijcp.12409, indexed in Pubmed: 24666884.

4. Ohlstein EH, von Keitz A, Michel MC. A multicenter, double-blind, randomized, placebo-controlled trial of the $\beta 3$-adrenoceptor agonist solabegron for overactive bladder. Eur Urol. 2012; 62(5): 834-840, doi: 10.1016/j.eururo.2012.05.053, indexed in Pubmed: 22695239.
5. Janicki JJ, Chancellor MB, Kaufman J, et al. Potential effect of liposomes and liposome-encapsulated botulinum toxin and tacrolimus in the treatment of bladder dysfunction. Toxins (Basel). 2016; 8(3), doi: 10.3390/toxins8030081, indexed in Pubmed: 26999210.

6. Di Rezze S, Frasca V, Inghilleri M, et al. Duloxetine for the treatment of overactive bladder syndrome in multiple sclerosis: a pilot study. Clin Neuropharmacol. 2012;35(5): 231-234, doi: 10.1097/WNF.0b013e3182613dce, indexed in Pubmed: 22751087.

7. Carbone A, Palleschi G, Conte A, et al. Gabapentin treatment of neurogenic overactive bladder. Pharmacol Exp Therap. 2014; 350: 543-551.

8. Lecci A, Maggi CA. Tachykinins as modulators of the micturition reflex in the central and peripheral nervous system. Regul Pept. 2001; 101(1-3): 1-18, indexed in Pubmed: 11495674.

9. Merrill L, Vizzard MA. Intravesical TRPV4 blockade reduces repeated variate stress-induced bladder dysfunction by increasing bladder capacity and decreasing voiding frequency in male rats. Am J Physiol Regul Integr Comp Physiol. 2014; 307(4): R471-R480, doi: 10.1152/ajpregu.00008.2014, indexed in Pubmed: 24965792.

10. Tyagi V, Philips BJ, Su R, et al. Differential expression of functional cannabinoid receptors in human bladder detrusor and urothelium. J Urol. 2009; 181(4): 1932-1938, doi: 10.1016/j.juro.2008.11.078, indexed in Pubmed: 19237176.

11. Wróbel A, RechbergerT. The Influence of Maxacalcitol, Vitamin D3 Analog, on Detrusor Overactivity in Conscious Rats. Urology. 2016; 93: 224.e7-224. e15, doi: 10.1016/j.urology.2016.03.008, indexed in Pubmed: 27018369.

12. Lecci A, Giuliani S, Meini S, et al. Nociceptin and the micturition reflex. Peptides. 2000; 21(7): 1007-1021, doi: 10.1016/s0196-9781(00)00241-2.

13. Rechberger T, Nowakowskit, Rechberger $\mathrm{E}$, et al Prevalence of common comorbidities among urogynaecological patients. Ginekol Pol. 2016; 87(5): 342-346, indexed in Pubmed: 27304649. 